\title{
The long term retention of levetiracetam in a large cohort of patients with epilepsy
}

\author{
C Depondt, A W C Yuen, G S Bell, T Mitchell, M J Koepp, J S Duncan, J W Sander
}

J Neurol Neurosurg Psychiatry 2006;77:101-103. doi: 10.1136/jnnp.2005.064626

Levetiracetam (Lev) is a new antiepileptic drug with a distinct mechanism of action, shown in regulatory trials to be effective. These controlled trials do not always predict how useful a drug will be in day to day clinical practice. Retention rates can provide a better indication of efficacy and tolerability in everyday use. Patients attending a tertiary referral centre for epilepsy and who received Lev in the first 2 years of its marketing were assessed $(n=811)$ to determine continuation rates of treatment with this drug. At the last follow up, $65 \%$ of patients were still taking Lev, and the estimated 3 year retention rate was $58 \%$. In total, $11 \%$ attained seizure freedom of at least 6 months. Patients taking greater numbers of concurrent antiepileptic drugs (AEDs) were more likely to discontinue Lev, and those reaching higher maximum daily dosages were less likely to discontinue Lev. The retention rate for Lev compares favourably with that of other new AEDs.

evetiracetam (Lev) is one of several antiepileptic drugs (AEDs) that have recently emerged for the adjunctive Lreatment of focal onset epilepsy. It was licensed as add on treatment for refractory focal epilepsy in adults in the UK in 2000. Lev is an S renantiomer pyr-olidone derivative with a distinctive pharmacological profile in animal models of seizures and epilepsy. ${ }^{1}$ It is not affected by drug-drug interactions and has a generally mild side effect profile. Lev was recently shown to act through binding to and modulation of the synaptic vesicle protein SV2A, ${ }^{2}$ which is thought to play a crucial role in vesicle function. Thus, Lev is fundamentally different from other AEDs, and its efficacy and tolerability profiles may also differ.

There are issues regarding the efficacy and safety of new AEDs, which have not been identified in regulatory trials. ${ }^{3}$ In total, 11 new AEDs have been licensed worldwide in the past 20 years, of which three (progabide, felbamate, and vigabatrin) developed safety problems, requiring prompt recall of patients to minimise morbidity. ${ }^{4}$ These problems developed after launch despite years of clinical trials, indicating the need for close monitoring of people exposed to such drugs, both to identify potential problems and to enable swift recall of patients. Additionally, it has transpired that some of these new AEDs have had little clinical impact, and now have only a small role in the treatment of people with epilepsy. It is important, therefore, that the clinical efficacy profile of these drugs in every day use is identified early, to instruct good clinical practice and thereby to improve the quality of life and wellbeing of people with epilepsy.

While the efficacy and tolerability of Lev have been demonstrated in several controlled regulatory trials, few studies have addressed long term continuation in a large group of patients in a clinic setting. This assessment aimed to determine the long term retention of treatment with Lev as a surrogate for efficacy and safety in a large group of patients in a single centre, to inform our future clinical practice in prescribing new AEDs.

\section{METHODS}

We identified all patients who were started on Lev within the first 24 months of marketing in the UK at the specialised epilepsy clinics of the National Hospital for Neurology and Neurosurgery (Queen Square and Chalfont Centre sites). This was part of an ongoing audit process and was approved by the local ethics committee. Patients who had Lev prescribed initially elsewhere or who had continued Lev after participating in a clinical trial were not included, to avoid survival bias. This assessment was carried out in our normal clinical settings and no additional procedures, visits or contact with patients were made. Follow up data were obtained from case notes.

The retention rate of Lev was estimated using KaplanMeier survival analysis. ${ }^{5}$ The effect of different factors on retention was examined using Cox regression analysis. ${ }^{6}$ Patients who discontinued Lev because of adverse events (AE) and inefficacy (IE) were considered separately; the 75 people who stopped because of both AE and IE were included in both estimations. We considered age at onset of epilepsy, age at start of treatment with Lev, gender, seizure type, number of concurrent AEDs, number of new AEDs (lamotrigine, topiramate, vigabatrin, or gabapentin) ever tried, initial dose of Lev, maximum recorded daily dose of Lev, titration schedule, and presence or absence of learning disability (LD). We calculated the number of patients attaining seizure freedom, the duration of seizure freedom, and the number of patients achieving a $\geqslant 50 \%$ reduction in seizure frequency.

\section{RESULTS}

Details of the 811 patients included are summarised in table 1. For 31 patients the duration of Lev therapy was unclear, and these patients were not included in the KaplanMeier and Cox estimations. Most of these (27/31) were still taking Lev when last reviewed.

At the last follow up, 528 patients $(65 \%)$ were continuing on Lev, and 3 year retention rate was estimated as $58 \%$ (fig 1). For those subjects taking at least one AED concomitant with Lev the retention rate was estimated at 56\%, and $53 \%$ for those taking at least two concomitant AEDs, although numbers were small. Only 39 patients started Lev as monotherapy, of whom $35(90 \%)$ were continuing Lev at last follow up.

Discontinuation was due to AE alone in 81 patients (10\%) and to $\mathrm{AE}$ and IE in 75 (9\%). The most frequent AEs were somnolence, dizziness, headache, behaviour problems, gastrointestinal disturbances, and skin rash. Patients taking

Abbreviations: $A E$, adverse events; $A E D$, anti-epileptic drug; IE, inefficacy; LD, learning disability; Lev, levetiracetam 


\begin{tabular}{|c|c|}
\hline Number of patients included & 811 \\
\hline \multicolumn{2}{|l|}{ Seizure type } \\
\hline Generalised seizures & $130(16 \%)$ \\
\hline Focal seizures & $517(64 \%)$ \\
\hline Mixed seizures & $155(19 \%)$ \\
\hline Seizure type unknown & $9(1 \%)$ \\
\hline \multicolumn{2}{|l|}{ Epilepsy syndrome } \\
\hline Cryptogenic focal epilepsy & $238(29 \%)$ \\
\hline Symptomatic focal epilepsy & $416(51 \%)$ \\
\hline Idiopathic generalised epilepsy & $68(8 \%)$ \\
\hline Other epilepsy syndrome & $68(8 \%)$ \\
\hline Not classifiable & $21(3 \%)$ \\
\hline Age, years; mean (range) & 37 (14 to 79$)$ \\
\hline Sex (Female/Male) & $410 / 401$ \\
\hline $\begin{array}{l}\text { Age at seizure onset, years; mean } \\
\text { (range) }\end{array}$ & 11 (0 to 63$)$ \\
\hline $\begin{array}{l}\text { With/without learning disability/ } \\
\text { not known }\end{array}$ & $164 / 493 / 154$ \\
\hline $\begin{array}{l}\text { Maximum recorded daily dose, } \\
\mathrm{mg} \text {; mean (range) }\end{array}$ & $2267\left(62.5^{*}\right.$ to 5000$)$ \\
\hline $\begin{array}{l}\text { Treatment time, months; mean } \\
\text { (range) }\end{array}$ & $16.7(0$ to 41$)$ \\
\hline \multicolumn{2}{|l|}{ Disposition: } \\
\hline Continuing at last follow-up & $528(65 \%)$ \\
\hline \multicolumn{2}{|l|}{ Stopped Lev } \\
\hline Adverse events only & $81(10 \%)$ \\
\hline Inefficacy only & $110(14 \%)$ \\
\hline Adverse events and inefficacy & $75(9 \%)$ \\
\hline Pregnancy & $3(0.4 \%)$ \\
\hline Deceased & $7(0.9 \%)$ \\
\hline Unknown & $7(0.9 \%)$ \\
\hline \multicolumn{2}{|l|}{ Concurrent AEDs at start } \\
\hline None & $39(5 \%)$ \\
\hline One & $253(31 \%)$ \\
\hline Two & $330(41 \%)$ \\
\hline Three & $151(19 \%)$ \\
\hline Four or more & $36(4 \%)$ \\
\hline Unknown & $2(0.2 \%)$ \\
\hline
\end{tabular}

greater numbers of concurrent AEDs were more likely to discontinue Lev due to AEs (for each extra concomitant AED taken, the hazard increased 1.2 fold (95\% confidence interval (CI) 1.03 to 1.44 ). Patients who reached higher maximum daily dosages were less likely to stop Lev because of $\mathrm{AE}$ (hazard ratio (HR) 0.38 ; $95 \%$ CI 0.31 to 0.46 ) for each additional $1000 \mathrm{mg}$ Lev per day). Patients with LD were 1.7 times more likely (95\% CI 1.19 to 2.43 ) to stop Lev because of AE than those without LD. Use of other new AEDs did not influence stopping Lev due to AE.

In total, 110 patients (13.6\%) stopped Lev because of IE alone, and 75 due to IE and AE. Patients taking greater numbers of concurrent AEDs were more likely to discontinue Lev due to IE (for each extra concomitant AED, the hazard increased 1.3 fold; $95 \%$ CI 1.11 to 1.51 ). Those who had tried more than two new AEDs were 1.6 times more likely (95\% CI 1.2 to 2.1) to stop Lev due to inefficacy than those who had taken $0-2$ of them. Patients who reached higher maximum daily dosages were significantly less likely to stop Lev due to IE (HR 0.69; 95\% CI 0.59 to 0.80 for each additional $1000 \mathrm{mg}$ Lev per day). All nine patients taking more than $4000 \mathrm{mg}$ continued Lev therapy. No effect was found for presence of LD.

Age at onset of epilepsy, age at start of treatment, starting dose, titration schedule, sex, and seizure type showed no clear effects for discontinuation due to AE or to IE.

Most subjects $(91 \%)$ had tried other new AEDs, either in the past or currently; $20 \%$ had taken one new AED, $25 \%$ had taken two, and $46 \%$ either three or four. All patients had tried at least one other AED prior to Lev.

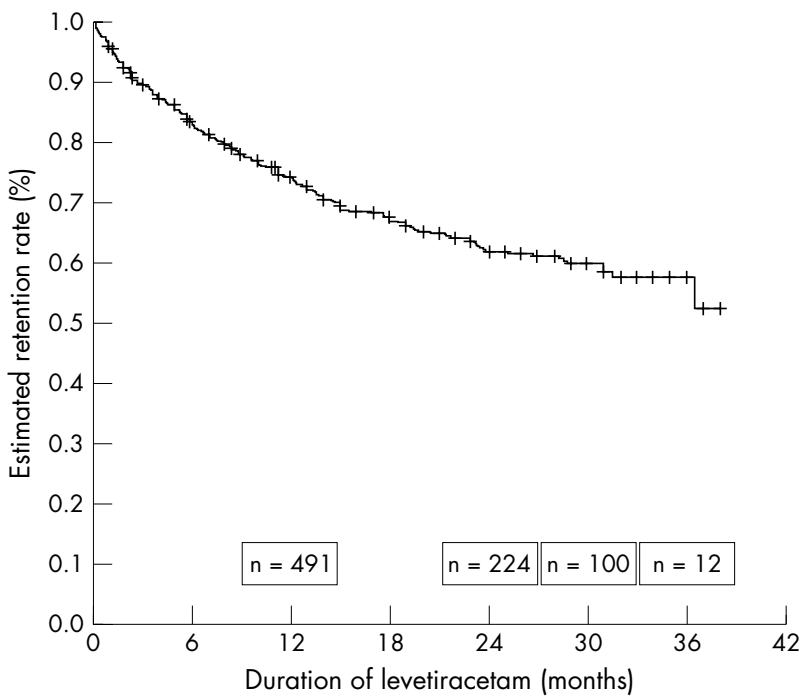

Figure 1 Estimated retention rate of levetiracetam in patients with epilepsy by Kaplan-Meier survival analysis. +Censored.

Seizure freedom was attained by $11 \%$ ( 89 patients) with a duration of 6-35 months (mean 16, median 15) and 54 patients had shorter periods of seizure freedom. Of 506 patients taking Lev for longer than 12 months 54 (11\%) were seizure free for at least a year. At least a further 237 patients $(29 \%)$ had a period of $\geqslant 50 \%$ reduction in seizure frequency. Seizure freedom of 6 months or more was attained in 72/654 (11\%) patients with cryptogenic or symptomatic focal epilepsy and in 13/68 (19\%) patients with idiopathic generalised epilepsy.

\section{DISCUSSION}

The efficacy and tolerability of Lev treatment in people with epilepsy have been demonstrated in several studies. ${ }^{7}$ Studies are often difficult to compare because of differences in study population and outcome measures.

In this assessment, the mean duration of follow up (16.7 months) is longer than in most previous studies. Retention rates are similar to those reported in other smaller studies. $^{8}$ The Kaplan-Meier analysis estimated a retention rate of $58 \%$ at 3 years, although numbers at this stage were small $(\mathrm{n}=12)$. We previously reported 3 year retention rates of $30 \%$ for topiramate, $29 \%$ for lamotrigine, and $<10 \%$ for gabapentin in patients recruited from the same epilepsy clinics. ${ }^{9}$ Discontinuation rates due to $\mathrm{AE}$ and IE for these three AEDs were 59, 56, and 76\% respectively, compared with $33 \%$ for Lev, suggesting that, in this setting, Lev is either better tolerated or more efficacious, or both. We observed a greater likelihood of discontinuing Lev with increasing numbers of concurrent AEDs and lower maximum daily dosage of Lev, similar to that observed with topiramate, lamotrigine, and gabapentin. Those with LD were more likely to discontinue Lev due to AE, suggesting a higher sensitivity to, or occurrence of, side effects in these subjects.

Reported figures for responder rates and percentage of patients achieving seizure freedom on Lev vary considerably amongst studies..$^{1011}$ Almost half our patients achieved a period of reduction in seizure frequency of $50 \%$ or more. A possible explanation for this tolerance and efficacy may be its distinct putative mechanism of action. Our results also provide some evidence that Lev may be efficacious both in patients with focal seizures, and in those with idiopathic generalised epilepsy. ${ }^{10}$

In summary, patients most likely to continue on Lev therapy are those without LD who are on few concurrent 
AEDs and able to tolerate relatively high dosages. We confirm the tolerability and efficacy of Lev in the treatment of chronic focal epilepsy; further studies are needed to confirm its efficacy in monotherapy and in generalised epilepsy. Finally, the favourable tolerability and efficacy profiles of Lev and its distinct putative mechanism of action hold promise for the development of a new category of AED.

\section{ACKNOWLEDGEMENTS}

We are grateful to S Sisodiya, $M$ Walker, $M$ Richardson, S Smith, M Trimble, P Hosking, M Mula, and A Russell for helping with the assessment of patients. The manufacturer of levetiracetam played no part in this assessment. The National Society for Epilepsy supported this work.

\section{Authors' affiliations}

C Depondt, A W C Yuen, G S Bell, T Mitchell, M J Koepp, J S Duncan, J W Sander, Department of Clinical and Experimental Epilepsy, UCL Institute of Neurology, and the National Hospital for Neurology and Neurosurgery, London, UK

Competing interests: our department has received research and travel grants from UCB Pharma, the manufacturer of levetiracetam. M J Koepp, J S Duncan and J W Sander have received honoraria for speaking at meetings sponsored by UCB. J W Sander was a paid member of an advisory panel to $U C B$.

Correspondence to: Professor Ley Sander, Department of Clinical and Experimental Epilepsy, National Hospital for Neurology and Neurosurgery, Queen Square, London WC1N 3BG, UK; Isander@ion. ucl.ac.uk
Received 1 February 2005

In revised form 31 March 2005

Accepted 28 April 2005

\section{REFERENCES}

1 Klitgaard H, Matagne A, Gobert J, et al. Evidence for a unique profile of levetiracetam in rodent models of seizures and epilepsy. Eur J Pharmacol 1998;353:191-206

2 Lynch BA, Lambeng N, Nocka K, et al. The synaptic vesicle protein SV2A is the binding site for the antiepileptic drug levetiracetam. Proc Natl Acad Sci USA 2004; 101:9861-6.

3 Walker MC, Sander JW. The impact of new antiepileptic drugs on the prognosis of epilepsy: seizure freedom should be the ultimate goal. Neurology, 1996:46:912-14.

4 Walker MC, Sander JW. Developments in antiepileptic drug therapy. Curr Opin Neurol 1994;7:131-9.

5 Kaplan EL, Meier P. Nonparametric estimation from incomplete observations. J Am Stat Ass 1958;53:457-81.

6 Cox DR. Regression models and life tables. JR Stat Soc 1972;34:187-220.

7 French JA, Kanner AM, Bautista J, et al. Efficacy and tolerability of the new antiepileptic drugs. II. Treatment of refractory epilepsy: report of the Therapeutics and Technology Assessment Subcommittee and Quality Standards Subcommittee of the American Academy of Neurology and the American Epilepsy Society, Neurology 2004;62:1261-73.

8 Nicolson A, Lewis SA, Smith DF. A prospective analysis of the outcome of levetiracetam in clinical practice. Neurology 2004;63:568-70.

9 Lhatoo SD, Wong IC, Polizzi G, et al. Long-term retention rates of lamotrigine, gabapentin, and topiramate in chronic epilepsy. Epilepsia 2000;41:1592-6

10 Betts T, Yarrow H, Greenhill L, et al. Clinical experience of marketed Levetiracetam in an epilepsy clinic-a one year follow up study. Seizure 2003; 12:136-40.

11 Morrell MJ, Leppik I, French J, et al. The KEEPER trial: levetiracetam adjunctive treatment of partial-onset seizures in an open-label community-based study. Epilepsy Res 2003;54:153-61. 